THE ASTROPHYSICAL JOURNAL, 518:866-872, 1999 June 20

(C) 1999. The American Astronomical Society. All rights reserved. Printed in U.S.A.

\title{
MASSES AND OTHER PARAMETERS OF THE POST-COMMON ENVELOPE BINARY BE URSAE MAJORIS
}

\author{
DONALD H. FERGUSON ${ }^{1}$ \\ Department of Physics, California State University at Hayward, Hayward, CA 94548; dhf@pacbell.net
}

JAMES LIEBERT ${ }^{1}$

Steward Observatory, University of Arizona, Tucson, AZ 85721; liebert@as.arizona.edu

\section{STEFAN HAAS ${ }^{2}$ AND RALF NAPIWOTZKI}

Dr. Remeis-Sternwarte Universität Erlangen-Nürnberg, Sternwartstrasse 7, 96049 Bamberg, Germany; mpai01@bamp01.sternwarte.uni-erlangen.de AND

THOMAS A. JAMES ${ }^{1}$

Departments of Mathematics and Science, Chaffey High School, Chaffey Joint Union High School District, Ontario, CA 91762;tajames@compuserve.com Received 1998 April 8; accepted 1999 January 2

\begin{abstract}
The binary star BE Ursae Majoris is recently emerged from the common envelope phase; indeed, the hot sdO/DAO component is the central star of the associated planetary nebula. As such, BE UMa represents an important test case of stellar evolution theory. Using the Hubble Space Telescope (HST) Goddard High Resolution Spectrograph (GHRS), we measured the radial velocity amplitude of the He II $\lambda 1640$ absorption line from the sdO/DAO component of this eclipsing system. Combining our results with those of Crampton, Cowley, \& Hutchings, we determine stellar masses in units of solar mass as follows: for the sdO, the mass is $0.70 \pm 0.07$, and that of the secondary star is $0.36 \pm 0.07$, where we report the $1 \sigma$ value for all errors. The separation between the component stars is $7.5 R_{\odot} \pm 0.5 R_{\odot}$ and is insensitive to small changes in inclination angle due to the near edge-on viewing angle of $84^{\circ} \pm 1^{\circ}$. Using these values, we modeled the eclipse light curve. Our results matched observed UBVR light curves of Wood and coworkers only if the modeled secondary star radius of $0.72 R_{\odot} \pm 0.05 R_{\odot}$ has nearly double the radius expected from the main-sequence mass-radius relation. The secondary star has thus not yet relaxed to thermal equilibrium since the common envelope phase ended $\sim 10^{4} \mathrm{yr}$ ago. Using the $\lambda 1640$ absorption-line profile and the surrounding continuum, we also were able to constrain the sdO helium abundance as $\log n_{\mathrm{He}}=-1.1 \pm 0.2$ and $\log n_{\mathrm{Fe}}<1$. Our results support the sdO/DAO $\log g \sim 6.5$ surface gravity and $T_{\text {eff }} \sim 100,000 \mathrm{~K}$ values of Liebert et al. and are consistent with the postAGB evolutionary track. Our best estimate of the distance to the BE UMa system is $2000 \mathrm{pc}$.

Subject headings: binaries: eclipsing — stars: abundances — stars: fundamental parameters stars: individual (BE Ursae Majoris) — stars: white dwarfs — ultraviolet: stars
\end{abstract}

\section{INTRODUCTION}

The nature of close binary stellar evolution is of general interest because the binary interaction may alter the evolution of the individual stellar components. Accretion onto a component which is at least partially degenerate is a wellknown characteristic of cataclysmic variables, X-ray binaries, bursters, and perhaps symbiotic stars. Of particular interest are situations where the mass transfer becomes unstable, such as when the mass donor is a red giant. The companion may be engulfed along with the core of the giant in a common envelope (CE) (for a review see Iben \& Livio 1993). This brief phase of stellar evolution is particularly difficult to model quantitatively, so a full understanding will be enhanced by more empirical data. That it may be occurring in many asymptotic giant branch stars prior to ejection of planetary nebulae is shown by the discoveries of 14 close binary planetary nuclei (PNNs; Bond 1994). However, the component parameters of these systems are

\footnotetext{
${ }^{1}$ Based on observations with the NASA/ESA Hubble Space Telescope, obtained at the Space Telescope Science Institute, which is operated by the Association of Universities for Research in Astronomy, Inc., under NASA contract NAS 5-2655.

${ }^{2}$ Current postal address: Goddard Space Flight Center, Code 681, Greenbelt, MD 20771.
}

generally poorly known and more analyses of such systems are needed. Only a few precataclysmic white dwarfs have measured masses: Hz 9 and Case 1 (Stauffer 1987), Feige 24 (Vennes et al. 1991; Vennes \& Thorstensen 1994), and the enigmatic V471 Tau, as observed by Sion et al. (1998) using the Hubble Space Telescope (HST).

BE UMa is a particularly interesting example of a PNN that has recently emerged from the CE phase. The primary is a very hot, high-gravity subdwarf $\mathrm{O}$ (or borderline DAO white dwarf) of surface effective temperature $\sim 100,000 \mathrm{~K}$, while the secondary is a late-type main-sequence star. BE UMa exhibits a unique continuum and emission-line spectrum, due to reprocessing of the hot UV continuum from the primary by the hemisphere of the secondary facing the primary. Fortuitously, BE UMa is also an eclipsing binary, providing a valuable constraint on the orbital inclination, so that precise mass determinations for the components are possible from radial velocity measurements. Like V471 Tau, however, the sdO/DAO absorption lines cannot be measured with sufficient precision from the ground, although for V471 Tau the contamination is due to the late-type $\mathrm{dK}$ star. Instead, we obtained high-quality UV spectra using the Goddard High Resolution Spectrograph (GHRS) aboard HST and used these and previously published emission-line radial velocities to determine masses of the 
BE UMa primary and secondary stars independently of any models.

Much progress has already been made in constraining the parameters of the BE UMa system. The system's variability was first noted by Kurochkin $(1964,1971)$. The blue continuum was discovered in the Palomar-Green Survey of Green, Schmidt, \& Liebert (1986), in which the star was referred to as PG $1155+492$. The UV-optical spectrum, which features a blue continuum and high-excitation emission lines, was first described in Ferguson et al. (1981), who found the spectrum to be cataclysmic variable-like but without the Doppler broadening characteristic of accretion disks. No flickering or polarization was noted. The current canonical model of the system was proposed by Ferguson (1981) and independently by Margon, Downes, \& Katz (1981). They postulated the existence of a very hot $T_{\text {eff }} \sim$ $100,000 \mathrm{~K} \mathrm{sdO} / \mathrm{DAO}$ primary whose extreme-ultraviolet (EUV) radiation generated a spectacular reflection effect at the facing hemisphere of the nearby main-sequence-like secondary star. Ferguson et al. (1981) found the radial velocity of the emission lines to be $K_{s}=95 \pm 7 \mathrm{~km} \mathrm{~s}^{-1}$. Margon et al. (1981) noted the continuum modulated with orbital phase, being brightest when the emission lines were most prominent.

Further work served to refine this model. Confirmation came from the timely discovery of Ando, Okazaki, \& Nishimura (1982), who found that the primary star of BE UMa was eclipsed by the secondary just when the optical emission and continuum spectrum was at minimum strength. Their observations limited the orbital inclination to $i \geq 84^{\circ}$, which constrained the mass function sufficiently to estimate mass. Unfortunately, the optical wavelength sdO/DAO absorption-line profiles are degraded by the reprocessed optical continuum and the emission lines, so that an additional assumption in lieu of radial velocities from both components was required. Most groups assumed the secondary star to be in thermal equilibrium and to lie on the main sequence, a postulate that we show below yields incorrect mass estimates. Crampton, Cowley, \& Hutchings (1983) observed BE UMa photometrically and spectroscopically. They found $K_{s}$ more precisely and observed a systemic radial velocity of $\gamma=-67 \pm 2 \mathrm{~km} \mathrm{~s}^{-1}$. They proposed a model in which the secondary star is a subgiant, which introduced the hypothesis that the secondary star might not be in thermal equilibrium. Hutchings \& Cowley (1985) used observations from $I U E$ to suggest that the blue continuum was variable at ultraviolet wavelengths. Such a result, if true, would cast serious doubt on the reprocessed secondary model. However, the data were also consistent with the object being variously off-center in the SWP aperture. Ferguson et al. (1987) provided an improved spectroscopic analysis, which included classifying the primary star based on line profiles obtained just before the primary star entered eclipse; i.e., when the reprocessed hemisphere of the secondary star was largely self-occulted. They also classified the secondary star, although these data were noisy Reticon spectra. Fulbright \& Liebert (1993) used CCD spectra of higher quality to estimate the spectral type of the secondary star, in which $\mathrm{TiO}$ bands were not seen, suggesting a spectral type of late K. Ferguson \& James (1994) quantitatively modeled the BE UMa reprocessed component. They found $\mathrm{C} / \mathrm{O} \sim 0.6$ and suggested that the $\lambda 4650$ blend feature usually attributed to $\mathrm{C}$ III or $\mathrm{N}$ III in cataclysmic variables $(\mathrm{CVs})$ is primarily recombinational $\mathrm{O}$ II. Their mass esti- mates were based on the secondary star lying on the mainsequence locus, however. Wood, Robinson \& Zhang (1995) observed BE UMa photometrically and provided the current best ephemeris: minimum light, or $\phi=0.00$, at HJD $2,444,998.2810+2.2911658(7) E$. They estimated $i>82.4$ \pm 0 . 1 , in close agreement with Crampton et al. (1983). They also provided mass estimates based on a main-sequence secondary star. Liebert et al. (1995) provided new primary star parameter estimates based on non-LTE models of the optical absorption-line profiles. They found $T_{\text {eff }}=105,000$ $\pm 11,000 \mathrm{~K}, \log \left(n_{\mathrm{He}} / n_{\mathrm{H}}\right)=0.1_{-0.2}^{+0.3}$, and $\log g=6.5 \pm 0.25$ for the primary star. They also obtained a deep $\mathrm{H} \alpha \mathrm{CCD}$ frame and discovered that $\mathrm{BE}$ UMa is embedded within a planetary nebula. The $[\mathrm{O} \mathrm{III}] \lambda 5007 \mathrm{CCD}$ imagery of $\mathrm{H}$. E. Bond (1996, private communication) displays the nebula more prominently. Finally, Ferguson et al. (1997) failed to detect $\mathrm{BE} \mathrm{UMa}$ at the shortest, least attenuated wavelengths observable with the EUVE, and concluded $n_{\text {metals }} / n_{\mathrm{H}}>0.01$ times solar to allow for sufficient quenching of the continuum due to metal ionization edges.

The sdO/DAO primary star is very hot and so dominates the observed energy distribution at UV wavelengths. The primary shows a hot Rayleigh-Jeans energy distribution with heavily pressure-broadened absorption lines of $\mathrm{He}$ II and $\mathrm{H}$ I. The secondary star reflection effect dominates at optical wavelengths, however, yielding a rich bound-free continuum and optical emission-line spectrum. In order to unambiguously observe the radial velocity shifts of the absorption feature with minimal contamination from the bound-free continuum of the secondary star reflection effect, we required spectra in the near-UV. In combination with radial velocity measurements of the emission-line component from our own HST/GHRS data and those of Crampton et al. (1983), and the tight constraints placed on orbital inclination by the eclipse of the sdO/DAO (Ando et al. 1982; Wood et al. 1995), we determine the mass function, and hence masses and binary separation, independent of any assumptions about secondary star spectral classification. We also use the absorption-line profile of $\mathrm{He}$ II $\lambda 1640$ to improve upon the precision of the sdO/DAO helium-to-hydrogen ratio.

\section{OBSERVATIONS}

We obtained HST/GHRS spectra of BE UMa at the He II $\lambda 1640$ line near both quadrature points and near orbital phase $\phi=0.50$, i.e., with the hemisphere of the secondary star illuminated by the sdO/DAO seen essentially face-on. The Small Science Aperture and the G160M grating were used in ACCUM mode, yielding an oversampled $0.069 \AA$ diode $^{-1}$. The spectra were substepped during integration, yielding a FWHM of 1.04 diode from measurement of several unsaturated wavelength calibration lamp emission lines.

Our data were reduced in standard fashion, except that special care was taken to ensure that precise radial velocity calibrations were made. Wavelength-dependent relative and absolute flux calibrations as described in Soderblom et al. (1995) were applied. Correction for Earth-orbital motion was also applied to each spectrum. We obtained wavelength calibration lamps immediately before and after each observation. Table 1 lists our observations. Column (1) shows the Space Telescope Science Institute (STScI) file number. Column (2) lists the midintegration times of each integra- 
TABLE 1

HST OBSERVATION LOG OF BE UMA AT $1640 \AA$

\begin{tabular}{cccc}
\hline \hline $\begin{array}{c}\text { STScI File } \\
\text { Number } \\
(1)\end{array}$ & $\begin{array}{c}\text { Midobservation } \\
(- \text { JD 2,450,000) } \\
(2)\end{array}$ & $\begin{array}{c}\text { Integration Time } \\
(\mathrm{s})\end{array}$ & $\begin{array}{c}\text { Orbital } \\
\text { Phase }^{\mathrm{a}} \\
(4)\end{array}$ \\
\hline z3f1a103t.c1h..... & 356.070536 & 979.2 & 0.456 \\
z3f1a203t.c1h..... & 356.703661 & 979.2 & 0.732 \\
z3f1a207t.c1h...... & 356.757649 & 2284.8 & 0.756 \\
z3f1a303t.c1h...... & 362.408954 & 979.2 & 0.222 \\
z3f1a307t.c1h..... & 362.462908 & 2284.8 & 0.246 \\
\hline
\end{tabular}

${ }^{\text {a }}$ At midintegration, using the ephemeris of Wood et al. 1995.

tion, column (3) shows the integration times, and column (4) shows the phase at midintegration based on the ephemeris of Wood et al. 1995.

The two spectra obtained at each quadrature were collected together, except that a wavelength calibration lamp was performed in between. We detected no instrumental wavelength shift between the two spectra collected near $\phi=0.25$, so these two were co-added without further processing. The two wavelength calibration lamps collected at either end of the short-exposure spectrum collected near $\phi=0.75$ (STScI file number z3f1a203t.c1h) showed a shift of $0.2 \AA$, perhaps due to thermal creaking of the telescope or the GHRS sometime during our observation. We averaged the two wavelength calibration lamps and applied the resulting wavelength scale to this spectrum. We measured no wavelength shift at all on either side of the longer of the two scans, however. The two spectra collected near $\phi=0.75$ were then co-added. The Digicon electron focusing introduced slight nonlinearities in all the measured wavelength scales. In order to conveniently analyze the data, we rebinned the three resulting spectra, one each at the quadrature points and near $\phi=0.50$, to wavelength scales of constant wavelength interval. Further analyses utilized these spectra. Figure 1 displays each such spectrum, but box-averaged across 13 pixels $(0.9 \AA)$ to average pattern

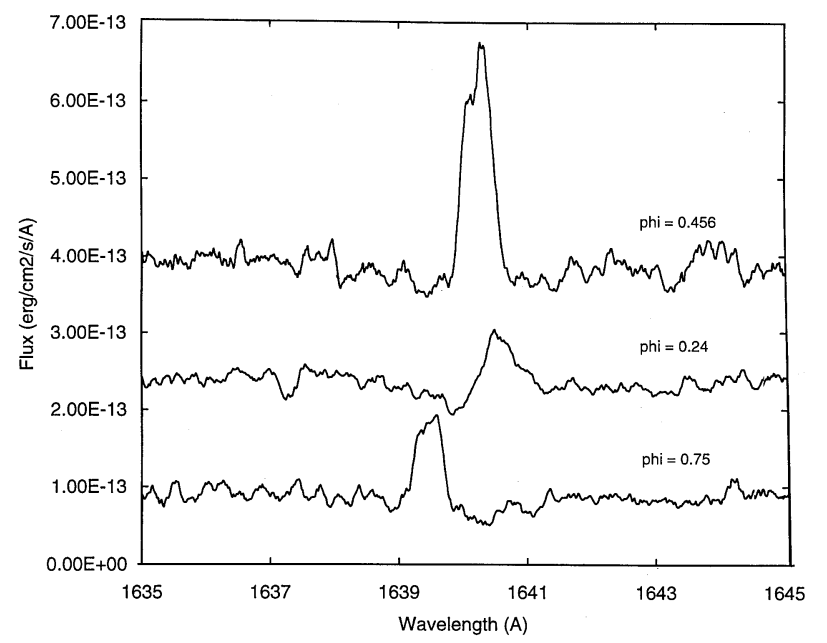

FIG. 1.-Reduced spectra box-smoothed over $0.9 \AA$ A The lowest spectrum shows the system at phase $\phi$ "phi" $=0.75$; the second spectrum, taken at $\phi=0.24$, is displaced upward by $f_{V}=1.5 \times 10^{-13} \mathrm{ergs} \mathrm{cm}^{-2} \mathrm{~s}^{-1}$, and the top spectrum obtained at $\phi=0.456$ is displaced upward by $f_{V}=$ $3.0 \times 10^{-13} \mathrm{ergs} \mathrm{cm}^{-2} \mathrm{~s}^{-1}$. Movement of the emission peaks is clearly seen in this double-line eclipsing system. The absorption lines are identifiable too but are better displayed in Fig. 2. noise. Note that the spectra are displaced vertically with respect to each other by $\Delta f_{v}=1.5 \times 10^{-13} \mathrm{ergs} \mathrm{cm}^{-2} \mathrm{~s}^{-1}$ $\mathrm{Hz}^{-1}$ for ease of viewing.

\section{MASS DETERMINATIONS AND HELIUM ABUNDANCE ESTIMATE}

As seen in Figure 1, each spectrum displays He II $\lambda 1640$ in both absorption and emission, which, in conjunction with the well-determined high-inclination orbit, allows the masses of the two components to be reliably determined. The absorption cores are generally partially obscured by the emission-line wings, however. The sdO/DAO component shows broad absorption lines, while the reflection effect exhibits emission lines of opposing radial velocity sense, in accord with the reflection effect model. We performed autocorrelations between the three combinations of pairs of spectra to measure the relative wavelength shifts between pairs and then computed the secondary radial velocity $K_{s}$ using equation (1) below:

$$
K=c\left(\lambda_{j}-\lambda_{i}\right) /\left\{\lambda\left[\sin \left(2 \pi \phi_{j}\right)-\sin \left(2 \pi \phi_{i}\right)\right]\right\} .
$$

Here $K$ is the radial velocity of either the primary star absorption lines or the secondary's emission lines (i.e., uncorrected as yet for the offset of the reflection effect from the secondary star's center); $\lambda_{i}$ and $\lambda_{j}$ are the central wavelengths for the $i$ th and $j$ th spectra obtained at orbital phases $\phi_{i}$ and $\phi_{j}$, respectively; and $c$ is the speed of light. We used the spectrum obtained near $\phi=0.50$ to obtain the zerovelocity wavelength $\lambda$.

We found $K_{s}=97 \pm 8 \mathrm{~km} \mathrm{~s}^{-1}$. Our standard deviation is computed purely on differences in $K_{s}$ values between the three pairs of HST data. Examination of Figure 1 shows one wing of the emission lines eroded by the absorption trough at quadrature; indeed, at " $\phi=0.456$," the emission line appears double-peaked owing to the underlying absorption trough. The autocorrelation technique minimizes the effect of this bias by considering the profile across the entire line while giving the highest weighting to the central, stronger portions of the emission line. Were our results significantly biased due to the presence of the absorption trough, our emission-line radial velocity measurements would be of significantly greater magnitude than results from a sample of less potential bias. Yet our findings are in close agreement with the earlier observations of Crampton et al. (1983), who measured $K_{s}=102 \mathrm{~km}$ $\mathrm{s}^{-1} \pm 2 \mathrm{~km} \mathrm{~s}^{-1}$. Their results were based on the optical emission-line profiles of hydrogen, which displays very thick damping wings and, owing to the dominance of the reflection effect bound-free continuum and relative intrinsic weakness of the very hot sDO/DAO hydrogen absorptionline profiles, a much weaker hydrogen line absorption component. We use their more precise value in our analysis.

The autocorrelation technique was less successful in isolating the absorption troughs because of their relatively shallower slopes and because of the potentially strong countervailing effects of the emission lines. We utilized spectra box-averaged over 5 diodes to reduce pattern noise, yielding a resolution of $0.35 \AA$ diode $^{-1}$ (still a much smaller wavelength interval than the absorption-line width) and located the bottom of the absorption trough in each spectrum. The uncertainty in the wavelength of the absorption trough position was found by computing the limits of the wavelength bands, i.e., the interval along the wavelength axis over which $1 \sigma$ statistical fluctuations in signal occurred 
in the continuum. Applying equation (1) again, we found the primary radial velocity to be $K_{p}=51 \pm 6 \mathrm{~km} \mathrm{~s}^{-1}$ based solely on statistical variations of the three data points. To explore the validity of this crucial value, we modeled the phase-dependent total profile. For the He II $\lambda 1640$ absorption-line component, we utilized the best-fit sdO/DAO profile of Liebert et al. (1995). To match the resolution of our data, we first convolved the absorptionline model with a Gaussian of 5 pixels. This profile closely matched the measured absorption wings, but the spectra were too noisy to estimate core depths. The emission component profile was taken from the nearby fully illuminated secondary measurement at $\phi=0.456$, and scaled sinusoidally with phase (cf. Margon et al. (1981); Ferguson \& James (1994). These components were shifted in wavelength according to their measured radial velocities, and the profiles were compared with the data of Figure 1. Except for noise effects, the match was near-perfect, including the relative strengths of the emission component. In particular, we found that the absorption cores at quadrature were deep enough to define the centers of absorption without any wavelength shift, even with emission-line infilling. One further check on the validity of our measurements was available. We used the phase-modulated emission- and absorption-line data to compute the system radial velocity. We found $\gamma=-60$ and $-72 \mathrm{~km} \mathrm{~s}^{-1}$ for the absorption and emission lines, respectively. This compares well with the more precise value of $\gamma=-67 \mathrm{~km} \mathrm{~s}^{-1}$ found by Crampton et al. (1983), and we note that the much more prominent emission-line peaks gave the same imprecision as our absorption-line measurements.

We next computed separation between the centers of gravity of each star. As Wood et al. (1995) have pointed out, the emission lines arise preferentially on the facing hemisphere of the companion, which is closer to the center of mass of the system than the center of mass of the secondary star. We integrated the first moment in displacement of the emission-line flux at orbital phase $\phi=0.75$. We used the specific intensity of Ferguson \& James (1994, eq. [6]). We found a mean offset of the emission-line surface toward the primary star of 0.424 of the secondary star radius. When the absolute value of this offset is added to the separation found using the emission-line radial velocities, the binary separation is found to be $a\left(R_{\odot}\right)=7.5 \pm 0.5$. In addition to the aforementioned errors in radial velocity computation, imprecision in orbital inclination of range $82.4 \leq i \leq 90^{\circ}$ was allowed for in the error computation.

We then computed the masses of the primary and secondary stars using the mass function. Our results are $M_{p}\left(M_{\odot}\right)=0.70 \pm 0.07$ and $m_{s}\left(M_{\odot}\right)=0.36 \pm 0.07$, where the errors represent 1 standard deviation.

We modeled the eclipse of the hot star using our own code and compared our results with the $U B V R$ photometry data of Wood et al. 1995. The high-gravity primary star was assumed to be spherical and was modeled by a square grid of length 100 squares across the equator and poles. Each square was assigned a temperature and specific intensity as determined by the Eddington approximation:

$$
\frac{I(0, \theta)}{I(0,0)}=\frac{2}{5}+\frac{3}{5} \cos \theta \text {. }
$$

Here limb darkening is expressed as the ratio of the specific intensity at any angle from the normal, as measured from the center of the star, to that of the normal value. We chose this relatively crude approximation because contamination from the reflection effect at the secondary star is known to exist even at eclipse minimum (cf. Ferguson et al. (1987)). For this reason, and because the secondary star contributes negligibly to the continuum except at $R$, the secondary star was treated as a disk of constant temperature. The primary star was then "moved" slowly behind the secondary star, and the combined continuum light curves at $U, B, V$, and $R$ wavelengths were generated, Values of stellar mass (and hence orbital speed), effective temperatures of both stars, radii of both stars, and orbital inclination were all varied to give a best-fit light curve at all wavelengths. Our results were highly sensitive to only two parameters: the orbital inclination and the secondary star radius. These sensitivities derived from the fact that the eclipse is a grazing one, and the primary star has a considerably smaller radius than the secondary star. That is, most of the eclipse duration is determined by the length of the chord of the path of the primary star across the secondary star. Yet the inclination is constrained by the known mass and surface gravity (and hence radius) of the primary. Unlike the situation in previous modeling attempts, availability of both stellar masses allowed the two stars' radii and temperatures, and the orbital inclination, to be free parameters. The uncertainties in derived parameters listed under "this paper" in Table 2, which summarizes the physical state of BE UMa, reflect the sensitivity and uniqueness of our fit to the model input values. They do not, however, take into account deviations from Eddington approximation limb darkening, which may be significant. For this reason, we prefer the measured primary star surface gravity estimate of Liebert et al. (1995), which, together with the primary star mass, yields the radius.

The highest possible signal-to-noise spectrum of the He II $\lambda 1640$ absorption feature was produced to facilitate fitting to sdO/DAO atmosphere models. The emission-line features were first removed from each spectrum. A band of width $1.2 \AA$ centered on each emission-line peak was removed. While this might seem a conservatively broad range (see Fig. 1, which is also smoothed over $0.9 \AA$ ), it ensured that no emission component remained in the data. The spectra were then box-averaged over 5 pixels, except within 5 pixels of the emission-line deadband. Here only the nonzero pixels outside the deadband were smoothed. The result is that the pattern noise remains intact very near the deadband in each spectrum. The two spectra obtained at quadrature were wavelength-shifted to match the orbital phase of the near-zero radial velocity observation obtained at $\phi=0.456$. The spectra were then averaged, with each spectrum weighted by its integration time. The result is shown in Figure 2. The small gap in the data at $\lambda 1640.0$ is due to an overlap of the emission-line deadband in all three spectra.

The surface helium content of the sdO/DAO primary provides information on the status of this star. Additionally, the determination of the fundamental parameters $T_{p}$, the primary star effective temperature, and $\log g$, the sdO surface gravity, from line profile fitting also depends moderately on the helium abundance. The determination of the helium abundance in Liebert et al. (1995) was hampered by the contamination of the inner region of the important He II $\lambda 4686$ line by the residual emission from the illuminated secondary. The profile of the He II $\lambda 1640$ line obtained with 
TABLE 2

Physical Characteristics of the BE UMa System

\begin{tabular}{|c|c|c|}
\hline Parameter & Value & Reference \\
\hline \multicolumn{3}{|l|}{ Subdwarf O star: } \\
\hline Mass $\left(M_{\odot}\right) \ldots$. & $0.70 \pm 0.07$ & This paper \\
\hline $\log$ sdO surface gravity $\ldots \ldots \ldots$ & $6.5 \pm 0.1$ & Liebert et al. 1995 \\
\hline Radius $\left(R_{\odot}\right) \ldots \ldots \ldots \ldots \ldots \ldots$ & $0.078 \pm 0.004$ & This paper \\
\hline Effective temperature $(\mathrm{K}) \ldots \ldots$ & $105,000 \pm 5000$ & This paper \\
\hline Helium abundance $. . . \ldots \ldots \ldots . .$. & $\log n_{\mathrm{He}}=-1.1 \pm 0.2$ & This paper \\
\hline Metallicity (low limit) ......... & $n_{\text {metals }} / n_{\mathrm{H}}>0.01$ times solar & Ferguson et al. 1997 \\
\hline Metallicity (high limit)... & $n_{\mathrm{Fe}}<10$ times solar & This paper \\
\hline \multicolumn{3}{|l|}{ Late-type dwarf star: } \\
\hline Mass $\left(M_{\odot}\right) \ldots \ldots \ldots$ & $0.36 \pm 0.07$ & This paper \\
\hline Radius $\left(R_{\odot}\right) \ldots \ldots \ldots$ & $0.72 \pm 0.05$ & This paper \\
\hline Effective temperature (K) & $5800 \pm 300$ & This paper \\
\hline Helium abundance & Solar-like & Ferguson \& James 1994 \\
\hline Metallicity & Solar-like, with $\mathrm{C} \sim 0.6 \mathrm{O}$ & Ferguson \& James 1994 \\
\hline \multicolumn{3}{|l|}{ Binary system: } \\
\hline Separation $\left(R_{\odot}\right)$ & $7.5 \pm 0.5$ & This paper \\
\hline Orbital period $\ldots \ldots \ldots \ldots \ldots \ldots$ & 2.2911658(E) HJD & Wood et al. (1995) \\
\hline Inclination $(\mathrm{deg}) \ldots \ldots \ldots \ldots \ldots$ & $84 \pm 1$ & This paper \\
\hline Distance $(\mathrm{pc}) \ldots \ldots \ldots$ & $\sim 2000$ & This paper \\
\hline Radial velocity $\left(\mathrm{km} \mathrm{s}^{-1}\right) \ldots \ldots$ & -67 & Crampton et al. 1983 \\
\hline
\end{tabular}

the GHRS allows for significant improvement in the precision of helium abundance determination.

The profile fit was performed with a set of non-LTE model atmospheres similar to that used in Liebert et al. (1995) but with slightly improved physics. We adopted hydrogen and helium mixtures. Detailed model atoms of hydrogen and helium were used, including a consistent treatment of Stark-broadened hydrogen and He II lines. We improved our model atmospheres by taking into account the pressure dissolution of the higher levels by the

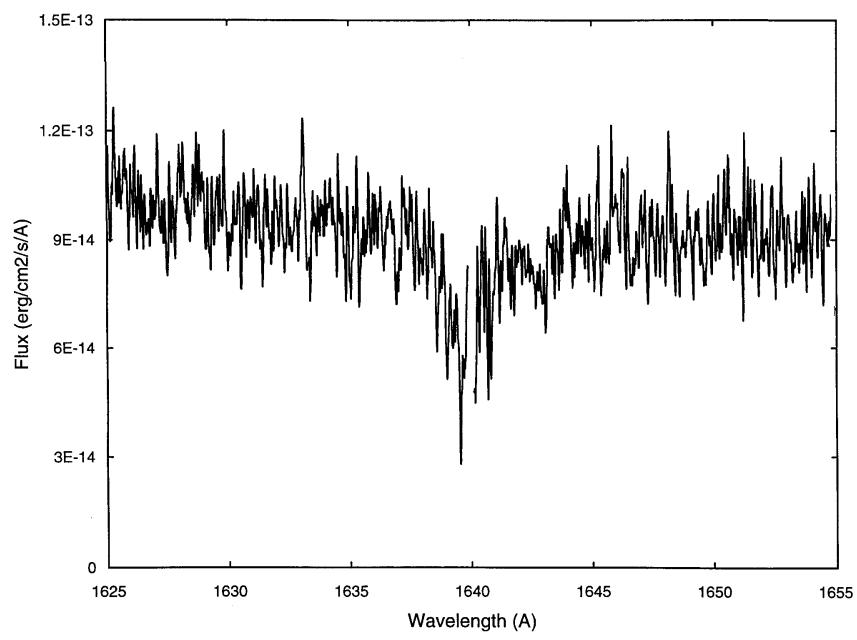

FIG. 2.-This spectrum is a composite made by removing a deadband of $\pm 0.6 \AA$ centered on the emission feature, shifting each spectrum in wavelength to match the $K_{p}=0.00 \mathrm{~km} \mathrm{~s}^{-1}$ case, and then co-adding the three spectra, which are weighted by integration time. The slight gap in data $1640.0 \AA$ is due to the presence of the emission deadband in all three spectra at this wavelength. Note that the composite spectrum signalto-noise ratio decreases near this wavelength because only one or two of the three absorption spectra without emission deadbands contribute. occupation probability formalism of Hummer \& Mihalas (1988). The non-LTE implementation is described in Hubeny, Hummer \& Lanz (1994). The He II broadening tables of Schöning \& Butler (1989) were used for the final profile calculation.

The He abundance was determined by line profile fitting of the He II $\lambda 1640$ line while we kept the effective temperature and surface gravity fixed at the values given in Liebert et al. (1995). We applied a slightly modified version of the least-squares fit algorithm developed by Bergeron, Saffer, \& Liebert (1992) and Saffer, Bergeron, \& Koester (1994). We used the calibrated flux observed and theoretical spectra and normalized both to a linear continuum. The synthetic spectra are convolved to the resolution of the GHRS spectrum with a Gaussian and interpolated to the actual parameters. The best fit was determined from a $\chi^{2}$ minimization. Dubious pixels were flagged out.

Following this procedure, we derived $\log n_{\mathrm{He}}=-1.1$ \pm 0.2 . The largest contribution to the error limits comes from the uncertainty of the effective temperature and $\log g$. The formal fit error is \pm 0.05 dex. This confirms the earlier results of Liebert et al. (1995). The great advantage of the new determination is a firm upper limit on the helium abundance. We can now definitely rule out any significant helium enrichment of the atmosphere. This abundance is not atypical of sdO/DAO stars before the onset of diffusion as the star becomes a white dwarf.

Additionally, the GHRS spectrum sets an upper limit to iron abundance in the hot primary. Here the dominant ionization species are $\mathrm{Fe}$ VI and Fe VII. While most of these lines are located shortward of $\lambda 1450$, a handful of Fe vi lines lie in the observed wavelength range in the vicinity of He II 21640. While an unambiguous iron abundance determination cannot be determined because of the weakness of these lines, an upper limit to iron abundance may be evaluated by comparing the observation with the predicted iron line strengths of models with differing iron contents. We 


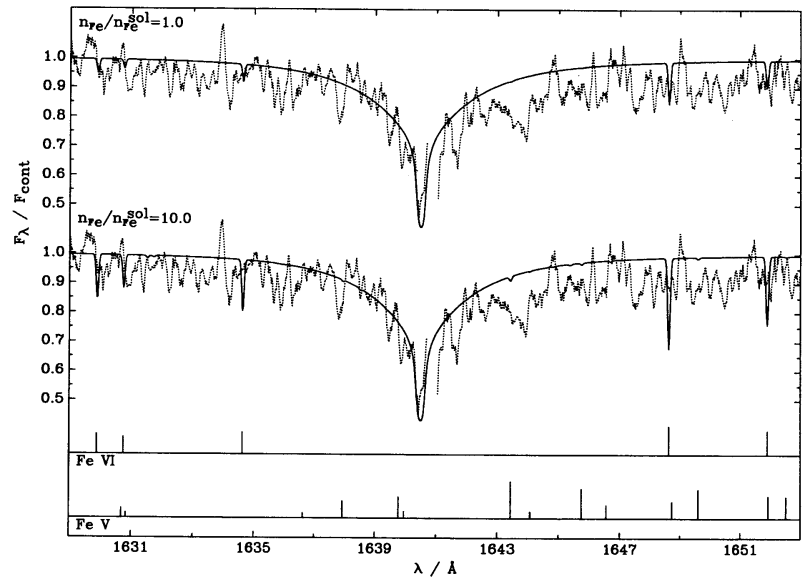

FIG. 3. - Comparison of a section of the GHRS spectrum of BE UMa near the $\mathrm{He}$ II $\lambda 1640$ line (dotted curve) with predictions from lineblanketed non-LTE models ( $T_{\text {eff }}=105,000 \mathrm{~K}, \log g=6.5$ ) with iron abundances of solar and 10 times solar (solid curves). Line positions of Fe $\mathrm{V}$ and $\mathrm{Fe}$ VI are given at the bottom with heights corresponding to $\log g f$-values.

computed a small grid of metal line-blanketed non-LTE models at $\log g=6.5$ and $T_{p}=105,000 \mathrm{~K}$. Besides $\mathrm{H}$ and $\mathrm{He}$, the chemical composition included $\mathrm{C}, \mathrm{N}$, and $\mathrm{O}$ together with generic ions representing simultaneously all the iron group elements from $\mathrm{Sc}$ through $\mathrm{Ni}$ (see Haas et al. 1996). The abundances of the light metals were always set to solar values for consistency with the solar-like metallicity found in the secondary (Ferguson \& James (1994). While relative ratios within the iron group were also kept solar, we varied the abundances of Sc through Ni by several dex. The resultant flux-normalized synthetic spectra were convolved with a Gaussian to the resolution of the GHRS instrument and compared with the observation. As absorption lines of $\mathrm{Fe}$ VI were not unambiguously visible, we conclude that iron abundance is less than 10 times solar, in accord with Ferguson et al. (1997). Figure 3 illustrates these conclusions for solar and 10 times solar iron abundances. Here the modeled curve is overlaid on the observed spectrum.

\section{EVOLUTION AS A POST-COMMON ENVELOPE/AGB BINARY}

The determination of the individual masses and the direct measurement of the mass ratio resolve some but not quite all ambiguities in the parameters of both stars.

The mass determination of $0.70 \pm 0.07 M_{\odot}$ is consistent with the conclusion favored by the discovery of a planetary nebula: that the BE UMa primary star evolved from the asymptotic giant branch as a $\mathrm{CO}$ degenerate core. However, the atmospheric parameters estimated by Liebert et al. (1995) fit to within their errors post-AGB tracks nearer $0.55 M_{\odot}$ (see Fig. 3 of that paper). Our inclination is to attribute this discrepancy to the well-known problem with $T_{\text {eff }}$ estimates from Balmer line fits to sdO, hot DA white dwarf and PNN stars (Napiwotzki 1992; Werner 1996). The temperature distribution of the upper atmosphere appears to be sufficiently affected by elements heavier than helium, so that non-LTE models assuming only hydrogen and helium often yield $T_{\text {eff }}$ values too low. This would result in fitting a post-AGB track too low in mass. We use the higher value of the primary's mass determined from the solution to the binary orbit instead of that inferred previously from the stellar atmospheres analysis. It is very important for what follows to note that the higher mass only strengthens the conclusion that the post-AGB evolutionary lifetime remains of the order of (a few times) $10^{4} \mathrm{yr}$, in at least approximate agreement with the likely kinematic age of the nebula.

The newly derived parameters of the secondary star now make it possible to show that its very recent immersion in a $\mathrm{CE}$ (i.e., $\sim 10^{4}$ yr ago) has affected its structure. Crampton et al. (1983) has concluded that the secondary was a subgiant, rather than a main-sequence star, in part because they assumed that the eclipse is total. Wood et al. 1995, in demonstrating that the eclipse is partial, argued that the secondary is only marginally larger than the expected radius near the end of its main-sequence phase.

These results show that the secondary has the mass of an $M$ main-sequence star, yet a radius and $T_{\text {eff }}$ quite inconsistent with such a low-mass main-sequence star. Using the relation of Lacey (1977) for $-1.0<\log \left(M / M_{\odot}\right)<+0.12$,

$$
\log \left(R / R_{\odot}\right)=0.917 \log \left(M / M_{\odot}\right)-0.020,
$$

we find the expected radius is $0.37 R_{\odot}$ for a secondary mass of $0.36 M_{\odot}$. Our inferred value is nearly twice this. The discrepancy cannot be explained by evolution to a larger radius during the main-sequence evolution (as discussed by Wood et al. 1995, though for a larger assumed mass). A 0.36 $M_{\odot}$ star is expected to have a main-sequence lifetime some 60 times the $10^{10} \mathrm{yr}$ of a $1.0 M_{\odot}$ star, assuming a mainsequence $L \sim M^{4}$. Such a star no older than the Galaxy should lie very near its ZAMS value. In any case, Lacey's empirical relation is based on measurements of $\mathrm{G}, \mathrm{K}$, and $M$ dwarfs and not the expected ZAMS radii. It is necessary to conclude that during its recent immersion in the CE, not only was the secondary out of thermal equilibrium, but its radius was substantially extended. Certainly $\sim 10^{4} \mathrm{yr}-$ the time since the AGB phase-is far less than the thermal timescale of the envelope of this star. Finally, we note that the period and separation of the binary are large enough that the secondary is not close to filling its Roche lobe and can be expected to be nearly spherical in shape (cf. Wood et al. 1995).

Likewise, the solar-like $T_{\text {eff }}$ estimate of $5800 \mathrm{~K}$ for the "back side" of the secondary, which we inferred essentially from its eclipse brightness, is perhaps also a factor of 2 larger than that expected for a $0.36 M_{\odot}$ main-sequence star. This temperature is very likely to be affected by the partial nature of the eclipse, the fact that the illuminated (and much warmer) hemisphere of the star is not completely shielded from view, and possibly by the transport of energy in the envelope of the star from the hotter to the cooler hemisphere. It is unlikely that the atmospheric temperature of the shielded hemisphere is really as high as $5800 \mathrm{~K}$ (appropriate to a $\mathrm{G}$ star), given the prior conclusions that the spectral type may be in the $\mathrm{K}$ range.

There remains an outstanding problem: If the $0.7 M_{\odot}$ primary star mass is correct and it fits a post-AGB track, the luminosity is high and the implied original mass is 3-4 $M_{\odot}$. For example, using the system masses and separation as collected in Table 1, assuming the primary star luminosity is the mean determined by Liebert et al. (1995), and adopting the formalism of Iben \& Tutukov (1993), we find the initial mass of the primary star to be $4.0 M_{\odot}$. This is also consistent, as Liebert et al. (1995) argued, with the kinematic age of the nebula matching the post-AGB track 
age of a few times $10^{4} \mathrm{yr}$, provided the primary is a postAGB star burning hydrogen in its shell. The evolutionary age is considerably older if it is burning helium instead.

The Iben \& Tutukov (1993) formalism depends upon knowing the luminosity of the primary star to good precision. Because of the problem discussed in Liebert et al. (1995), encountered when fitting the primary star spectrum to hydrogen-line models, our luminosity estimate is not precise to better than a factor of 2 or so. Further, our error bars do not preclude a primary mass of $0.6 M_{\odot}$.

The above scenario appears to be inconsistent with the system being well above the Galactic plane, out in the halo. Even thick disk stars are older and therefore of lower mass. There are B stars and runaway $\mathrm{O}$ stars observed tens of kiloparsecs from the plane, so apparently young objects do exist out in the halo. Perhaps the gas cloud from which the binary was formed was ejected early on from the disk.

There is evidence from one other case that $\mathrm{CE}$ evolution can somehow leave remnants of higher than expected mass from low initial mass primary stars. Bond et al. (1999) report that the V471 Tau primary star has a mass of 0.85 $M_{\odot}$. This is higher than the derived masses for all the other white dwarfs (all but one are single stars) in the Hyades, yet the white dwarf is the hottest in the cluster. One would expect the last white dwarf formed to be lowest in mass if the initial/final mass relation were monotonic. For example, if we assume that the primary star fits the standard postAGB models, such as those of Vassiliadis \& Wood (1994), we have the dilemma of a theory implying a massive, and therefore young, progenitor primary star. Of course, V471 Tau has followed a very different evolutionary path from the single stars, but still the formalism of Iben \& Tutukov
(1993) predicts more mass loss through the CE phase, producing a remnant of lower mass than in the single-star case.

\section{SUMMARY}

Double-line spectroscopy of the BE UMa eclipsing binary system, particularly measurements of the elusive sdO/DAO using the He II $\lambda 1640 \mathrm{UV}$ absorption line, has yielded the masses of the sdO/DAO and secondary stars. They are 0.70 and $0.36 M_{\odot}$, respectively. The absorption profile of the He II $\lambda 1640$ line also places a firm upper limit to surface helium abundance of the sdO/DAO primary: $\log n_{\mathrm{He}}=-1.1$. We used these results to model the light curve, and found the secondary star radius to be $0.72 R_{\odot}$.

Results from this HST study thus determine important parameters of the components in the BE UMa system. The interpretation of the primary as a post-AGB star consistent with its planetary nebula and massive enough to produce a $\mathrm{CO}$ white dwarf is made more secure. It is the secondary star that offers what is perhaps a unique view of the nature of an object just removed from a CE. We have shown here that it has a radius bloated to twice the value of the radius of a main-sequence star of its mass, and must be well away from thermal equilibrium.

The authors thank the staff of the STScI for their observing plan support and responsible data reduction aid. D. H. F. and J. L. acknowledge support from NASA HST grant GO-6427. S. H. acknowledges support from the Deutsche Agentur für Raumfahrtangelegenheiten (DARA) under grants 50 QV 97026 and 50 QV 97054. We also thank the anonymous referee for useful comments.
Ando, H., Okazaki, A., \& Nishimura, S. 1982, PASJ, 34, 141

Bergeron, P., Saffer, R. A. \& Liebert, J. 1992, ApJ, 394, 228

Bond, H. E. 1994, in Interacting Binary Stars, ed. A. W. Shafter (San Francisco: ASP)

Bond, H. E, Schaefer, K. G., Sion, E. M., Saffer, R., \& Cheng, F. H. 1999, in Proc. 11th European Conf. on White Dwarfs, ed. J. E. Solheim (San Francisco: ASP), in press

Crampton, D., Cowley, A. P., \& Hutchings, J. B. 1983, ApJ, 272, 202

Ferguson, D. H. 1981, presentation at the Santa Cruz Cataclysmic Variable Conference

Ferguson, D. H., \& James, T. A. 1994, ApJS, 94, 723

Ferguson, D. H., Liebert, J., Cutri, R., Green, R. F., Willner, S. P., Steiner, J. E., \& Tokarz, S. 1987, ApJ, 316, 299

Ferguson, D. H., Liebert, J., Green, R. F., McGraw, J. T., \& Spinrad, H. 1981, ApJ, 251, 205

Ferguson, D. H., Leibert, J., Napiwotzki, R., \& Haas, S. 1997, AJ, 114, 1227

Fullbright, M. S., \& Liebert, J. 1993, ApJ, 410, 275

Green, R. F., Schmidt, M., \& Liebert, J. 1986, ApJS, 61, 305

Haas, S., Dreizler, S., Heber, U., Jeffrey, S., \& Werner, K. 1996, A\&A, 311, 669

Hubeny, I., Hummer, D. G., \& Lanz, T. 1994, A\&A, 282, 151

Hummer, D. G., \& Mihalas, D. 1988, ApJ, 331, 794

Hutchings, J. B., \& Cowley, A. P. 1985, PASP, 97, 328

\section{REFERENCES}

Iben, I. Jr., \& Livio, M. 1993, PASP, 105, 1373

Iben, I. Jr., \& Tutukov, A. V. 1993, ApJ, 418, 343

Kurochkin, N. E. 1964, Perem. Zvezdy, 15(1), 7 1971, Perem. Zvezdy, 18, 85

Lacey, C. H., 1977, ApJS, 34, 479

Liebert, J., Tweedy, R., Napiwotzki, R., \& Fulbright, M. S. 1995, ApJ, 441, 424

Margon, B., Downes, R. A., \& Katz, J. I. 1981, Nature, 293, 200

Napiwotzki, R. 1992, in Lecture Notes in Physics 401, The Atmospheres of Early-Type Stars, ed. U. Heber \& C. S. Jeffrey (Berlin: Springer), 310

Saffer, R. A., Bergeron, P., \& Koester, D. 1994, ApJ, 351, 366

Schöning, T., \& Butler, K. 1989, A\&AS, 78, 51

Sion, E. M., Schaefer, K. G., Bond, H. E., Saffer, R. A., \& Cheng, F. H. 1998, ApJ, 496, L29

Soderblom, D. R., Gonella, A., Hulbert, S. J., Leitherer, C., Schultz, A., \& Sherbert, L. E. 1995, Instrument Handbook for the Goddard High Resolution Spectrograph (GHRS) (Baltimore: STScI)

Stauffer, J. R. 1987, AJ, 94, 996

Vassiliadis, E., \& Wood, P. R. 1994, ApJS, 92, 125

Vennes, S., Thorstensen, J., Thejll, P., \& Shipman, H. L. 1991, AJ, 72, L37

Vennes, S., \& Thorstensen, J. 1994, AJ, 108, 1881

Werner, K. 1996, ApJ, 457, L39

Wood, J. H., Robinson, E. L., \& Zhang, E. M. 1995, MNRAS, 277, 87 